\title{
Relative drift between black aurora and the ionospheric plasma
}

\author{
E. M. Blixt ${ }^{1,3}$, M. J. Kosch ${ }^{2,4,5}$, and J. Semeter ${ }^{3}$ \\ ${ }^{1}$ Department of Physics, University of Troms $\varnothing$, N-9037 Troms $\emptyset$, Norway \\ ${ }^{2}$ Space Vehicles Directorate, Air Force Research Laboratory, Hanscom AFB, Massachusetts, USA \\ ${ }^{3}$ Department of Electrical and Computer Engineering, Boston University, USA \\ ${ }^{4}$ On leave from Communication Systems, Lancaster University, UK \\ ${ }^{5}$ Honorary Research Fellow, University of Kwazulu-Natal, Durban, South Africa \\ Received: 6 December 2004 - Revised: 12 May 2005 - Accepted: 12 May 2005 - Published: 27 July 2005 \\ Part of Special Issue "Atmospheric studies by optical methods"
}

\begin{abstract}
Black auroras are recognized as spatially welldefined regions within uniform diffuse aurora where the optical emission is significantly reduced. Although a well studied phenomenon, there is no generally accepted theory for black auroras. One theory suggests that black regions are formed when energetic magnetospheric electrons no longer have access to the loss cone. If this blocking mechanism drifts with the source electron population in the magnetosphere, black auroras in the ionosphere should drift eastward with a velocity that increases with the energy of the precipitating electrons in the surrounding aurora, since the gradient$B$ curvature drift is energy dependent. It is the purpose of this paper to test this hypothesis. To do so we have used simultaneous measurements by the European Incoherent Scatter (EISCAT) radar and an auroral TV camera at Troms $\varnothing$, Norway. We have analyzed 8 periods in which a black aurora occurred frequently to determine their relative drift with respect to the ionospheric plasma. The black aurora was found to drift eastward with a velocity of $1.5-4 \mathrm{~km} / \mathrm{s}$, which is in accordance with earlier observations. However, one case was found where a black patch was moving westward, this being the first report of such behaviour in the literature. In general, the drift was parallel to the ionospheric flow but at a much higher velocity. This suggests that the generating mechanism is not of ionospheric origin. The characteristic energy of the precipitating electron population was estimated through inversion of $E$-region plasma density profiles. We show that the drift speed of the black patches increased with the energy of the precipitating electrons in a way consistent with the gradient- $B$ curvature drift, suggesting a magnetospheric mechanism for the black aurora. As expected, a comparison of the drift speeds with a rudimentary dipole field model of the gradient- $B$ curvature drift speed only yields order-ofmagnitude agreement, which most likely is due to the nightside disturbed magnetosphere being significantly stretched.
\end{abstract}

Keywords. Auroral ionosphere; MI interaction; Energetic particles, precipitating

\section{Introduction}

Black auroras are easily recognized as relatively small regions with a distinct reduction of auroral luminosity, within large-scale regions of otherwise homogeneous diffuse aurora. Two distinctly different types of black auroras have been identified. The most commonly observed are black patches, which exhibit a variety of forms and, mapped to an altitude of $105 \mathrm{~km}$, they are typically around $5 \times 1 \mathrm{~km}$ in extent (Trondsen and Cogger, 1997; Kimball and Hallinan, 1998a). The second type are black vortex streets, which have a clockwise rotation (opposite to that of auroral curls), and commonly have a wavelength of about $1-3 \mathrm{~km}$ (Trondsen and Cogger, 1997; Kimball and Hallinan, 1998b). Both types are most common in the midnight sector in the late substorm recovery phase. The visual appearance of the two types is dramatically different, basically because black patches show no signs of shear. While black patches move predominantly in the eastward direction with a speed of 1 to $4 \mathrm{~km} / \mathrm{s}$ (all the black patches of Parsons and Thomas (1973); SchouteVanneck et al. (1990) and Trondsen and Cogger (1997) were drifting eastward), black vortex streets exhibit a large spread in both drift direction and speed. Black patches have also been found to have much longer lifetimes than the vortex structures. Black arcs, typically around $0.5 \mathrm{~km}$ thick (Trondsen and Cogger, 1997), are an intermediate state which can be difficult to classify. This is foremost because both black patches and vortex streets often are formed from black arcs, but also because the drift direction of an east-west aligned arc with smooth boundaries is difficult to estimate. In this paper we focus on the drift of black patches. 
The generation mechanism of black auroras is uncertain. Two major mechanisms have been proposed over the years:

1. An active ionospheric mechanism where a downward field-aligned current associated with a positive space charge is pulling cold electrons out of the E-region (Royrvik, 1976; Marklund et al., 1997; Trondsen and Cogger, 1997). This mechanism has predominately been associated with black vortex streets.

2. A magnetospheric mechanism that locally hinders the strong pitch angle diffusion/scattering into the loss cone, and thus stops electrons from precipitating (Kimball and Hallinan, 1998a; Peticolas et al., 2002; Blixt and Kosch, 2004).

We refer to the latter as a blocking mechanism, although it can be argued that it is not an active blocking mechanism, but merely a region where the strong pitch angle diffusion/scattering is not enabled. However, the fact that pitch angle diffusion is enabled in the large area covered by the diffuse aurora, and that black regions within the diffuse aurora are very small and coherent structures, justifies our choice of terminology.

The relative drift between the black aurora and the ionospheric plasma can provide us with important information regarding the source mechanism. The generally most accepted theory of black vortex streets associates them with a Kelvin-Helmholtz instability due to shear in the $\boldsymbol{E} \times \boldsymbol{B}$ velocity induced by an excess positive charge. As such, the vortices must move with the plasma flow (Keskinen and Ganguli, 1996; Marklund et al., 1997; Kimball and Hallinan, 1998b). It is expected that auroral features associated with field-aligned currents move more or less with the $\boldsymbol{E} \times \boldsymbol{B}$ flow in the E-region, although a relative drift of the aurora with respect to the background plasma of around $100 \mathrm{~m} / \mathrm{s}$ has been observed (Haerendel et al., 1993). This relative drift velocity is found to increase during the expansion phase of a substorm and for bright aurora (del Pozo et al., 2002b). The relative drift is thought to arise from parallel electric fields which decouple the ionosphere from the magnetosphere and the acceleration region, a mechanism referred to as braking field lines by Haerendel et al. (1993). So in the case of black aurora, where the parallel electric field is expected to be small, or directed downwards, the relative drift should be expected to be small.

On the other hand, it has been suggested that black auroras are due to structures in the hot electron population which gradient- $B$ curvature drift around the Earth (Kimball and Hallinan, 1998a). If these structures drift along with the energetic plasma, the black patches should follow the drift of the hot magnetospheric electron population. This drift velocity is energy dependent, so we are interested in the relationship between the energy of the precipitating electrons, which surround the black patches, and the ionospheric drift velocity of the black aurora. By combining radar and optical data of the black aurora, it becomes possible to study this relationship.
We begin in Sect. 2 by describing the instrumentation used in the study. Then in Sect. 3 we discuss the observations and the drift speeds we have observed in the black aurora. In Sect. 4 we explain how the characteristic energy of the auroral particles have been obtained, and we establish a positive correlation between the characteristic energy of the auroral particles and the black auroral drift speed in Sect. 5. The main results and conclusions are summarized in Sect. 6.

\section{Instrumentation}

The EISCAT (European Incoherent Scatter facility) mainland UHF radar consists of three antennae; the transmitter, located in Troms $\varnothing$, Norway $\left(69.58^{\circ} \mathrm{N}, 19.22^{\circ} \mathrm{E}\right)$, and two receiving antennae, one in Kiruna, Sweden $\left(67.86^{\circ} \mathrm{N}, 20.44^{\circ} \mathrm{E}\right)$ and one in Sodankylä, Finland $\left(67.36^{\circ} \mathrm{N}, 26.63^{\circ} \mathrm{E}\right)$. In the tristatic mode, each antenna measures the line-of-sight velocity of the ion population in a given common volume. For a common volume in the F-region $(\sim 300 \mathrm{~km})$, where the collisional drift is negligible compared to the $\boldsymbol{E} \times \boldsymbol{B}$ drift, a good estimate of the horizontal local $\boldsymbol{E} \times \boldsymbol{B}$ plasma drift is obtained. Post-integration of the radar data (a basic integration of 5-10 s is typical) is needed to reduce the noise in the measurements. The radar data from the March 2002 events were post-integrated for $30 \mathrm{~s}$, while 20-s integration was used for the radar data from March 2003. However, to obtain the tristatic velocity estimates from the radar data, it was necessary to perform additional post-integration, to a total of $300 \mathrm{~s}$, to obtain enough signal-to-noise ratio. The March 2002 experiment was "tau3". This has an initial time resolution of $5 \mathrm{~s}$ and an unambiguous height resolution of $5.4 \mathrm{~km}$. The March 2003 experiments were "arc1", which have a subsecond time resolution and a range resolution of about $1 \mathrm{~km}$. The first range gate was around $92 \mathrm{~km}$ for both experiments.

To optically identify and track the black patches, the digital auroral imager, Odin, from the University of Troms $\varnothing$ was used. The imager consists of a GEN III, extended blue, image intensified (XYBION ISS-750) camera. A F0.95 lens with $14.35 \times 10.92^{\circ}$ field of view (yields a $26.4 \times 21.1 \mathrm{~km}$ view at $105 \mathrm{~km}$ altitude), observing in white light, was used in all observations. The video stream is digitized in real time, and converted to (PAL) DV, and a GPS controlled time stamp synchronizes the imager to the radar. The imager was located close to the Troms $\varnothing$ antenna $(<500 \mathrm{~m})$, and pointed up along the magnetic field line direction, parallel to the radar beam. To derive the velocity of the black patches, we have identified the center of form of the structures, which is analogous to the center of mass of the black structures if the black pixels had been given a mass. These center of forms have been tracked as they traverse the field of view. The black patches can be tracked for approximately $10-15 \mathrm{~s}$ before they change their shape too much or disappear out of view. Longer measurement times yield better velocity estimates. The distance the center of form moves is simply calculated by mapping the auroral luminosity to an altitude of $105 \mathrm{~km}$. This choice of altitude follows from the plasma 
Table 1. Overview of events analyzed in this paper. $K$ is the local 3-h averaged activity index.

\begin{tabular}{lccll}
\hline Name & Date \& Time [UT] & $K$ & MLT sector & Substorm phase \\
\hline & $2002-03-05$ & & & \\
02A & $20: 03: 20-20: 05: 00$ & 5 & pre-midnight & Growth/quiet \\
02B & $20: 12: 58-20: 14: 46$ & 5 & pre-midnight & Growth/quiet \\
02C & $20: 21: 20-20: 22: 34$ & 5 & pre-midnight & Growth/quiet \\
& & & & \\
& $2003-03-02$ & & & \\
03A & $22: 01: 10-22: 04: 10$ & 6 & midnight & Expansion \\
03B & $22: 04: 30-22: 07: 30$ & 6 & midnight & Expansion \\
03C & $23: 52: 20-23: 57: 20$ & 6 & post-midnight & - \\
& & & & \\
& $2003-03-04$ & & & Recovery/onset \\
03D & $22: 57: 30-22: 58: 20$ & 5 & post-midnight & Recovery/onset \\
03E & $23: 09: 30-23: 13: 40$ & 5 & post-midnight & Recold \\
\hline
\end{tabular}

density measurements shown in Figs. 7 through Figs. 10, if we assume the peak in electron density coincides with the peak of ionization (peak luminosity). The error in the velocity estimate varies linearly with the error in the altitude estimate, which is less than $10 \%$, as estimated from the peak of the electron density profile. The velocity estimate of the perturbations traveling along the border of the black aurora (the ripple drift) is less certain. However, we are not seeking any quantitative measure of the ripple drift in this paper, instead we are merely interested in it as a qualitative indicator of the behavior of the black aurora, as there is reason to believe that sheared black auroras are different to simple black patches and arcs (see discussion in Blixt and Kosch (2004), Kimball and Hallinan (1998a) and Kimball and Hallinan (1998b)).

The Kilpisjärvi IRIS imaging riometer $\left(69.05^{\circ} \mathrm{N}\right.$, $20.79^{\circ} \mathrm{E}$ ) operates at $38.2 \mathrm{MHz}$, and monitors the relative cosmic noise absorption in 49 different beam directions. The absorption maximizes at around $90 \mathrm{~km}$ altitude and below, which corresponds to precipitating electrons with characteristic energy of a few tens of $\mathrm{keV}$ to a few hundred $\mathrm{keV}$ (del Pozo et al., 2002a). The effective field of view at $100 \mathrm{~km}$ altitude is a square which covers $67.8^{\circ}-70.2^{\circ}$ latitude and $17.8^{\circ}-23.8^{\circ}$ east longitude, so the field line which crosses the EISCAT common volume at $300 \mathrm{~km}$ altitude is well within the field of view.

\section{Observations}

The data presented in this paper stem from two observational campaigns, one in March 2002 and one in March 2003, where EISCAT was run in a tristatic mode and the common volume was monitored through the Odin imager. A typical observation of black patches, with the EISCAT plasma drift velocity measurement superposed, is shown in Fig. 1 (this one from 2 March 2003, 22:02:08 UT).

Table 1 gives the geophysical context for each black aurora event in the two campaigns. The substorm phase has

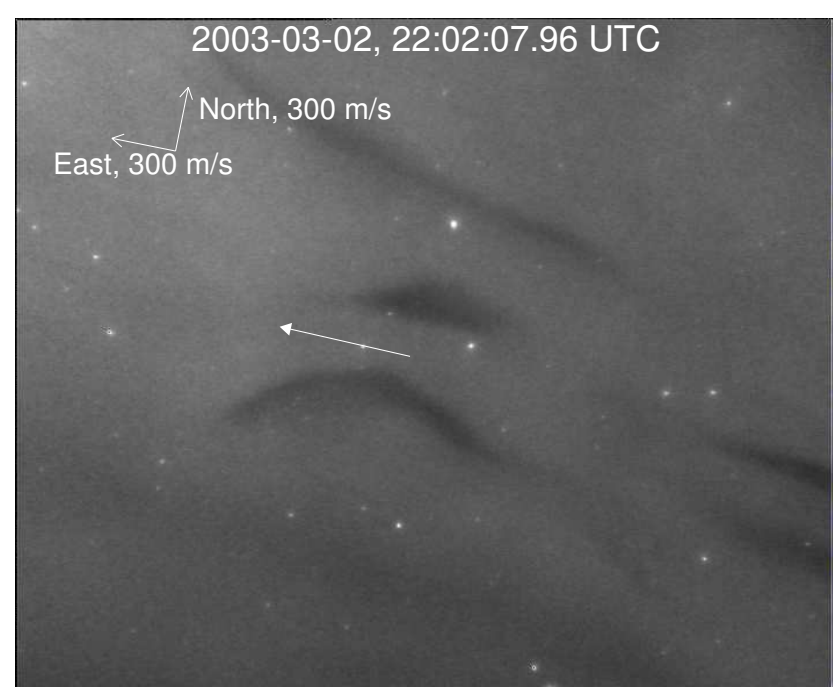

Fig. 1. An example image of the black aurora from event 03A. The velocity measured from the EISCAT radar is shown as a vector near the center. Note how the black structures are aligned with the plasma velocity vector.

been determined using the University of Troms $\varnothing$ 's magnetometer chain in northern Scandinavia. The March 2002 events (02A-02C) happened just after a substorm, during rather quiet conditions. The March 2003 events (03A-03E) all happened in substorm expansion phase or right in between substorms, and exhibit much more active auroras than the 2002 events. This is also seen in the electron density measurements. For March 2002 (02A-02C) the electron density rarely exceeds $10^{11} \mathrm{~m}^{-3}$ (Figs. 7 and 8), while it is significantly higher in the other events (Figs. 9 and 10). There were no indications of plasma density dropouts in the E-region associated with any of the black aurora events, which Blixt and Kosch (2004) used as an indicator for the lack of strong downward field-aligned currents when they analyzed events 


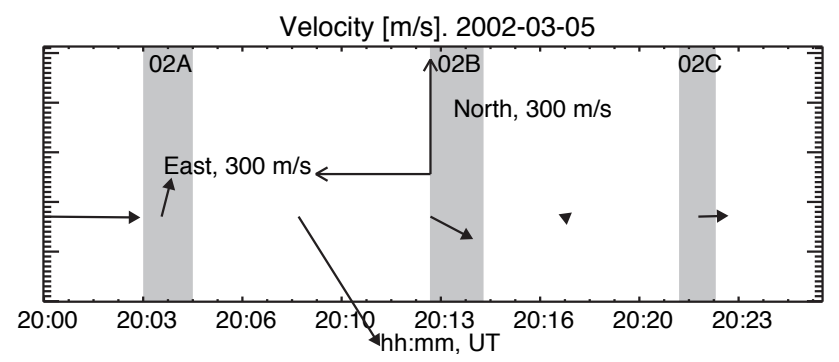

Velocity [m/s]. 2003-03-02

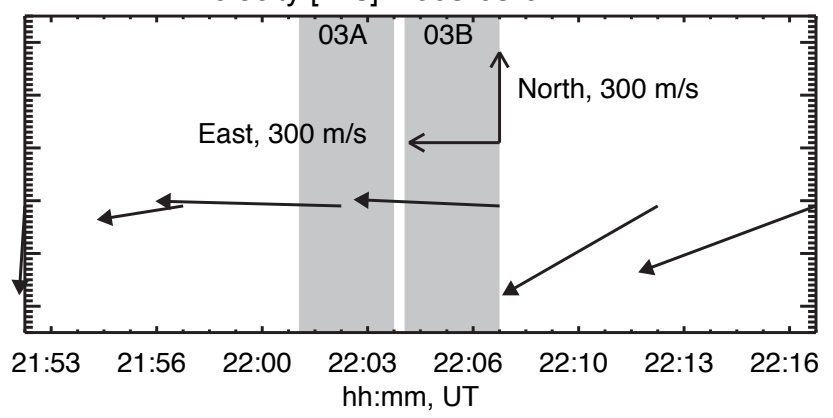

Velocity [m/s]. 2003-03-02

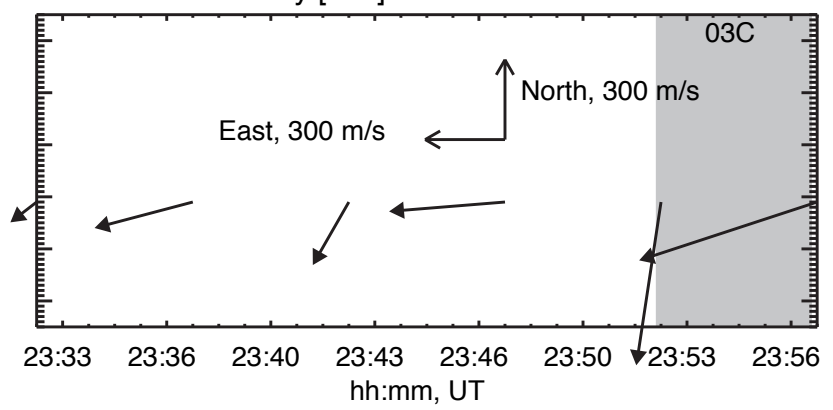

Velocity $[\mathrm{m} / \mathrm{s}] .2003-03-04$

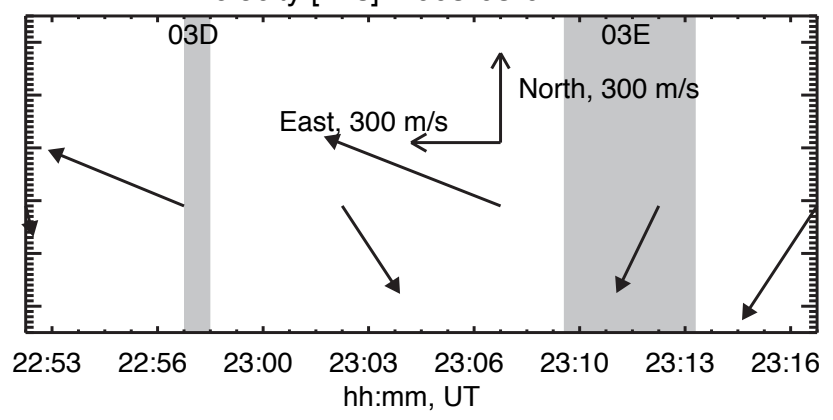

Fig. 2. Summary of the plasma drift measurements by EISCAT. The black aurora observations are indicated by the gray areas and are labeled according to Table 1.

02A and 02B. For the black aurora in events 03A-03D, the motion through the radar beam is too fast for this conclusion to be tested. Figure 2 shows the ionospheric plasma flow measured by the tristatic EISCAT radar. East is to the left and north is up, as if the plasma flow were seen from below. The general westward flow direction and speed in events $02 \mathrm{~A}, 02 \mathrm{~B}$, and $02 \mathrm{C}$, is consistent with being in the pre-midnight sector. Events $03 \mathrm{~A}-03 \mathrm{E}$ all occur in the mid-
Table 2. Visual characteristics of each event.

\begin{tabular}{ll}
\hline Event & Notes \\
\hline 02A & 2 black arcs drift S. ripples move W. \\
02B & Black patches evolve into thin black arc, drifting N. \\
02C & Thin distorted black arcs, short-lived drift W. \\
03A & Black elongated patches drifting W, then E. \\
03B & Black arc dissolves, patches drifts E, some shear. \\
03C & Black elongated patches in puls. aurora, drifting E, \\
& then SE \\
03D & Thin elongated black patches moving E \\
03E & Black arc with shear and pulsating aurora.
\end{tabular}

night sector, or later, and these events all show a predominantly eastward flow. All flow measurements are consistent with SuperDARN observations, except for 02A, where no SuperDARN data is available.

Table 2 summarizes the main visual characteristics of each event, such as drift direction of the black aurora, whether there were perturbations drifting along on the borders of the black region (ripples), and if the auroras have any shear flow. Video clips from all the events can be viewed at http://www. phys.uit.no/ $\sim$ maarten/Results/AnnGeo05.html.

The March 2002 events (02A and 02B) were analyzed by Blixt and Kosch (2004), and the black aurora from event 02A is shown there. In event 02A two east-west aligned black arcs drift slowly south, and fast moving ripples were seen to move west along the borders of the black arcs. In event 02B, two black patches slowly transform into a thin black arc, which drifts slowly northward. Ripples are seen to move both east and west along the borders. For both of these events there is no connection between the drift of the optical black aurora and the plasma flow, and there is no sign of shear flow in the aurora. Event $02 \mathrm{C}$ shows thin distorted black arcs intertwined with streaks of enhanced luminosity on a diffuse background. Black arcs are seen to drift west at the end of the event, which correlate well in both direction and magnitude with the $\boldsymbol{E} \times \boldsymbol{B}$ flow.

In event $03 \mathrm{~A}$ the black structures drift much faster than in the earlier events. An example image of the black aurora during this event is shown in Fig. 1. At the beginning several black patches drift westward at a speed of about $2.6 \mathrm{~km} / \mathrm{s}$, directly anti-parallel to the observed plasma flow. The black patches then slow down and start moving eastward, with a slightly lower speed. This is the only report of westward drifting black patches in the literature as far as the authors know. Figure 1 shows the black patches just as they are starting to move eastward. Event $03 \mathrm{~B}$ is the direct continuation of $03 \mathrm{~A}$, and covers a period where the diffuse aurora gradually disintegrates. The lower image in Fig. 3 shows how the diffuse aurora is nearly gone at the end of the event. At the beginning there are two thin black arcs (not shown) aligned with the $\boldsymbol{E} \times \boldsymbol{B}$ flow vector. The two black arcs soon separate and disappear, and there are signs of shear flow in the 

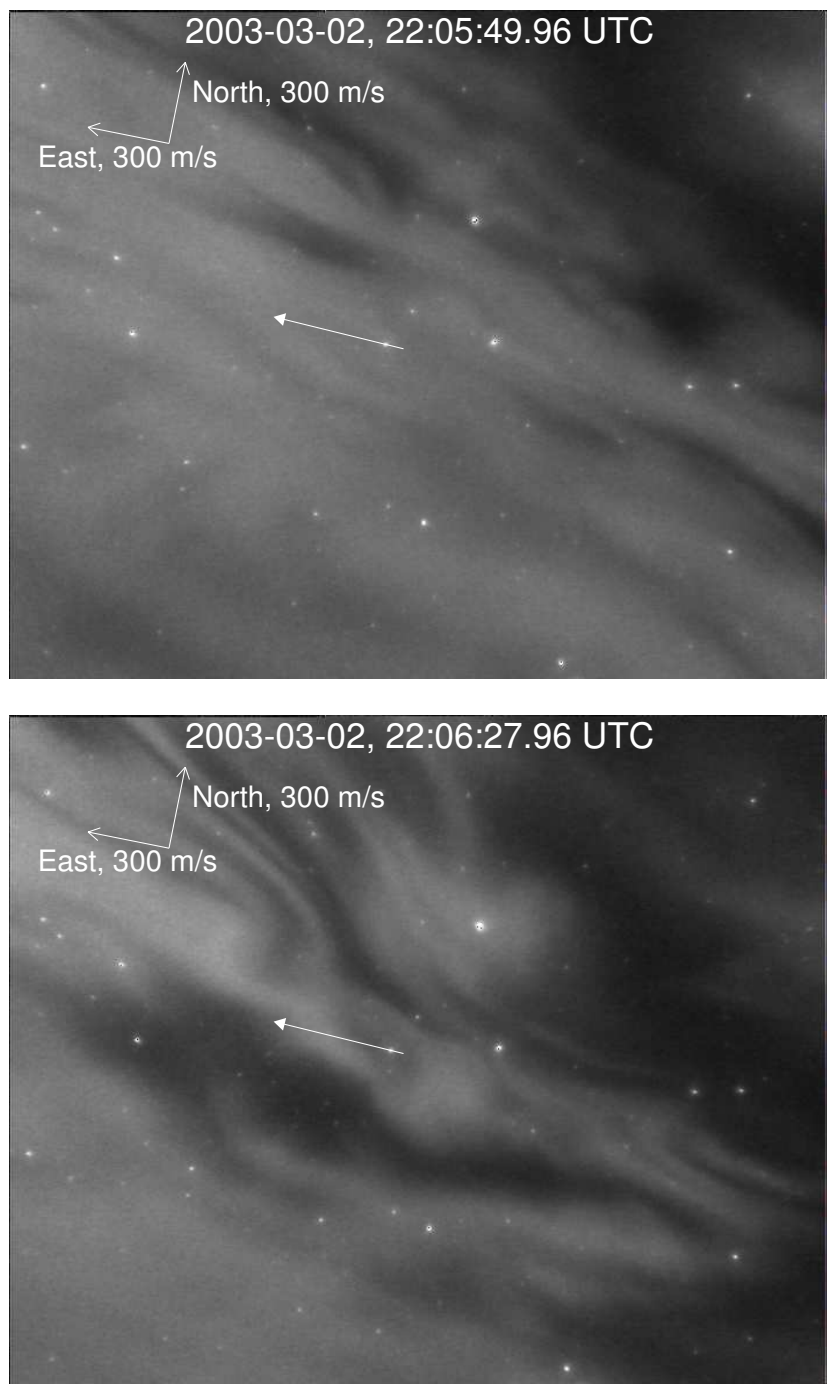

Fig. 3. Two images from event $03 \mathrm{~B}$, showing the difference in the structure of the black aurora in the middle of the event (upper image) and closer to the end (lower image), where the diffuse aurora is nearly gone. In the upper image one of the black patches used for tracking the drift velocity is seen just above and left of the center.

process. In the middle of the event, several black patches appear which move eastward (with $1.5-2.7 \mathrm{~km} / \mathrm{s}$ ) parallel to the measured plasma flow (one of the black patches can be seen just left and above the center of the upper image in Fig. 3). At the end of this sequence one can not safely identify the black aurora, as there seems to be a turbulent mixing of diffuse aurora with non-luminous parts of the sky (lower image in Fig. 3).

Event $03 \mathrm{C}$ happens about an hour later in the postmidnight/early morning sector and the electron density has decreased significantly.

In the diffuse aurora (which showed large pulsating patches) there was an east-west aligned black arc, with a sharp bend towards north, which showed no clear drift. The pulsating aurora partially covers the black arc so that veiling occurs (Kimball and Hallinan, 1998a). In Fig. 4, the upper
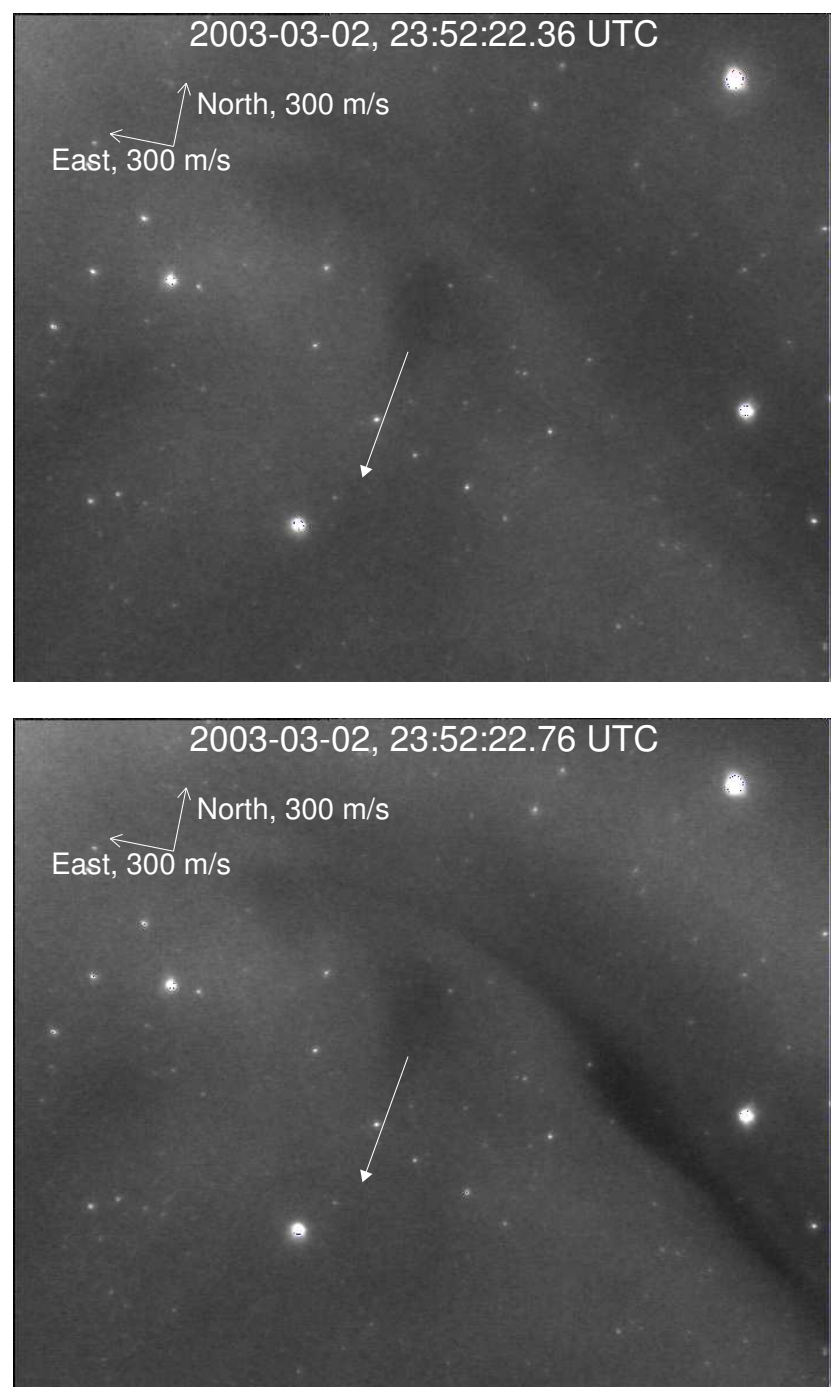

Fig. 4. Two images from event $03 \mathrm{C}$, about $0.5 \mathrm{~s}$ apart, showing the black aurora in an on (upper image) and off (lower image) phase of the pulsating aurora (veiling).

image shows the pulsating aurora covering the black aurora. Half a second later, the lower image in Fig. 4 shows how the black aurora has reappeared again. About $20 \mathrm{~s}$ into the event, the black arc transforms into several elongated black patches which are seen to drift eastward.

Event $03 \mathrm{D}$ occurred in an active period. Thin elongated black patches were seen to drift rapidly eastward (around $4 \mathrm{~km} / \mathrm{s}$ ). The drift is remarkably well aligned with the measured plasma flow, as can be seen in Fig. 5, but much faster than the plasma flow.

Ten minutes later event 03E starts. The plasma flow had slowed down slightly, being more towards the southeast. Two parallel black arcs were visible, southeast to northwest aligned, but strong pulsating aurora partially covered the black aurora, which made drift velocity estimates difficult. Figure 6 shows the two black arcs and how the pulsating aurora on phase (lower image) covers the southernmost 


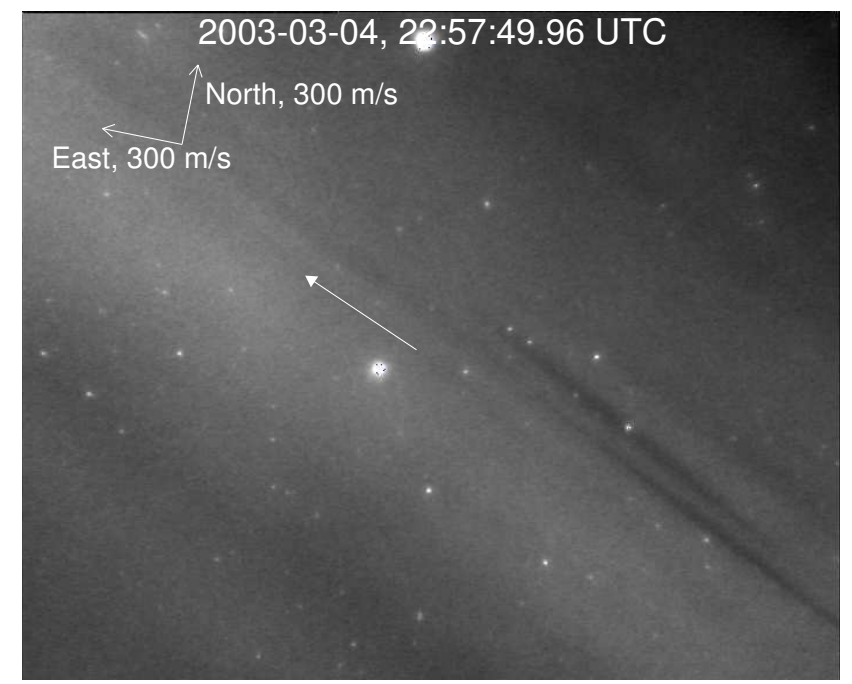

Fig. 5. An image from event $03 \mathrm{D}$, where elongated black patches were drifting rapidly and parallel to the plasma flow (indicated by the vector).

Table 3. Comparison of the different velocities observed. $v_{p}$ is plasma flow velocity measured by the EISCAT radar, $v_{d}$ is the drift velocity of black structures, $v_{r}$ is the velocity of small-scale perturbations or ripples on the borders of the black structures. NW stands for northwest, etc.

\begin{tabular}{llll}
\hline Event & $v_{p}[\mathrm{~km} / \mathrm{s}]$ & $v_{d}[\mathrm{~km} / \mathrm{s}]$ & $v_{r}[\mathrm{~km} / \mathrm{s}]$ \\
\hline $02 \mathrm{~A}$ & $0.5 \mathrm{NW}-0.3 \mathrm{SW}$ & $0.05 \mathrm{~S}$ & $2 \mathrm{~W}$ \\
$02 \mathrm{~B}$ & $0.6 \mathrm{SW}-0.1 \mathrm{~S}$ & $0.05 \mathrm{~N}$ & $0.8 \mathrm{E}$ and $\mathrm{W}$ \\
$02 \mathrm{C}$ & $0.7 \mathrm{~W}$ & $0.6 \mathrm{E}$ and $\mathrm{W}$ & - \\
$03 \mathrm{~A}$ & $0.6 \mathrm{E}$ & $1.6 \mathrm{E}-2.6 \mathrm{~W}$ & - \\
$03 \mathrm{~B}$ & $0.5 \mathrm{E}$ & $1.5-2.7 \mathrm{E}$ & $4 \mathrm{E}-6 \mathrm{~W}$ \\
$03 \mathrm{C}$ & $0.7 \mathrm{SE}$ & $1.5 \mathrm{SE}-2.2 \mathrm{E}$ & - \\
$03 \mathrm{D}$ & $0.5 \mathrm{NE}$ & $4 \mathrm{E}$ & - \\
03E & $0.3 \mathrm{SE}$ & - & $7 \mathrm{E}$ \\
\hline
\end{tabular}

black arc. At times, the pulsations covers both arcs. Later in the event, the northernmost black arc became visible for several seconds, and fast moving perturbations on its northern boundary were seen.

Table 3 summarizes the plasma flow measurements of the radar and the observed black auroral drift velocities for each event. The riometer observations tabulated in Table 4 indicate that there are precipitating particles with energies over $25 \mathrm{keV}$ present in all events. As a part of the tail of the electron energy distribution, this is consistent with the assumed altitude of the background aurora $(105 \mathrm{~km})$ within which the black aurora is visible, as well as the typical precipitating particle energy associated with diffuse aurora (Paschmann et al., 2002).
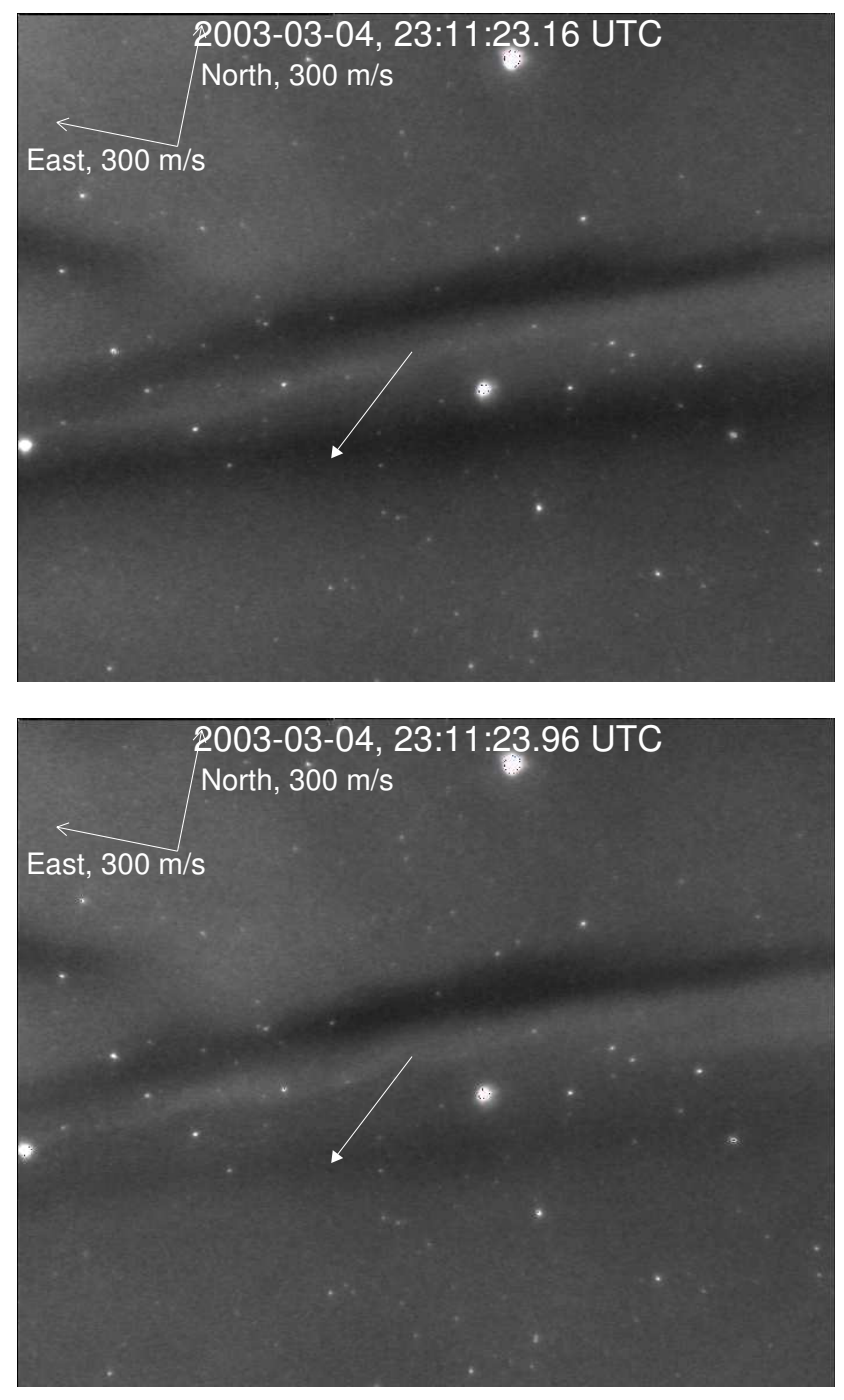

Fig. 6. Two images from event 03E, less than one second apart, showing the off (upper image) and on (lower image) phase of a pulsating aurora which partially covers the southernmost black aurora.

\section{Analysis}

Our goal is to determine whether a relationship exists between the drift velocity of black aurora and the characteristic energy of the causative electron flux in the surrounding diffuse aurora. The electron density profiles measured by EISCAT provide indirect information about the characteristic energy and the overall shape of the incident electron energy spectrum. As the characteristic energy increases, the altitude of maximum electron density decreases in a predictable manner. Models of the ionospheric response to an auroral particle source have been used to formulate an inverse problem whereby the differential number flux spectrum may be estimated from a measured $E$-region density profile with no prior assumption on its functional form (Vondrak and Baron, 1975; Brekke et al., 1989). Semeter and Kamalabadi (2005) have developed a variant implementation of this procedure involving the Maximum Entropy method and have 
Table 4. Summary of the absorption data from the IRIS imaging riometer. The fitting parameter $(\gamma)$, the estimated characteristic energy $\left(E_{0}\right)$ and temperature $(k T)$, from Eq. (1) is shown for each event.

\begin{tabular}{lcccc}
\hline Event & Absorption $[\mathrm{dB}]$ & $\gamma$ & $E_{0}[\mathrm{keV}]$ & $k T[\mathrm{keV}]$ \\
\hline 02A & $\sim 0.3$ & 1.2 & 2.7 & 2.6 \\
02B & $\sim 0.3$ & - & - & - \\
02C & $\sim 0.3$ & 0.6 & 1.7 & 1.7 \\
03A & $\sim 0.4$ & 1.1 & 5.5 & 4.9 \\
03B & $\sim 0.5$ & 4.0 & 9.5 & 2.6 \\
03C & $\sim 0.2$ & 1.5 & 6.6 & 3.8 \\
03D & $\sim 0.2$ & 1.0 & 9.5 & 7.6 \\
03E & $\sim 0.5$ & 0.8 & 11.5 & 8.5 \\
\hline
\end{tabular}

investigated its sensitivity to the principle sources of uncertainty in the model and measurements. Their procedure was found to be robust in the following sense. When an ionospheric electron density profile was computed using a given measured electron energy spectrum, the original spectrum could be reliably recovered, even if large statistical errors were incorporated in the measurement. The bulk properties in the recovered spectrum (e.g. net energy flux, characteristic energy) were also reliably recovered in the presence of certain types of model uncertainty. Direct verification of inversion results is difficult owing to the difficulty in obtaining in-situ measurements for small-scale auroral forms. However, Kirkwood and Eliasson (1990) and Osepian and Kirkwood (1996) have demonstrated order-of-magnitude agreement with conjugate satellite measurements.

Figures 7 through 10 show typical electron density profiles, together with the accompanying recovered spectra for each event. Some cautionary points should be made concerning the interpretation of the recovered spectra. First, as the electron energy increases, the sensitivity of the altitude of the ionization peak to changes in characteristic energy decreases dramatically. At the same time, for energies $<500 \mathrm{eV}$, the electron deposition altitude is above $180 \mathrm{~km}$, where electron density may be influenced by horizontal transport. Thus, there is a range of energies from 0.5 to $\sim 40 \mathrm{keV}$ where the inversion can be considered reliable.

Although the shape of the recovered energy spectra is not constrained in the inversion, the spectra often take the form of an exponentially decaying background distribution with an additional suprathermal distribution (bump in tail), as seen in Figs. 7 to Figs. 10. Extensive studies show that the $\kappa$ distribution is very good at fitting the high energy tail often found in space plasmas (Vasyliunas, 1968; Olsson and Janhunen, 1998; Kletzing et al., 2003). In our effort to fit the recovered spectra to a model, we choose to use a simpler distribution closer to the Maxwellian distribution,

$F(E)=A_{0}(E / k T)^{\gamma} \exp \left(-\frac{E}{k T}\right)$
Time: 2002-03-05, 20:03:29 UTC, $\mathrm{E}_{0}=2.7[\mathrm{keV}], \mathrm{kT}=2.6[\mathrm{keV}]$
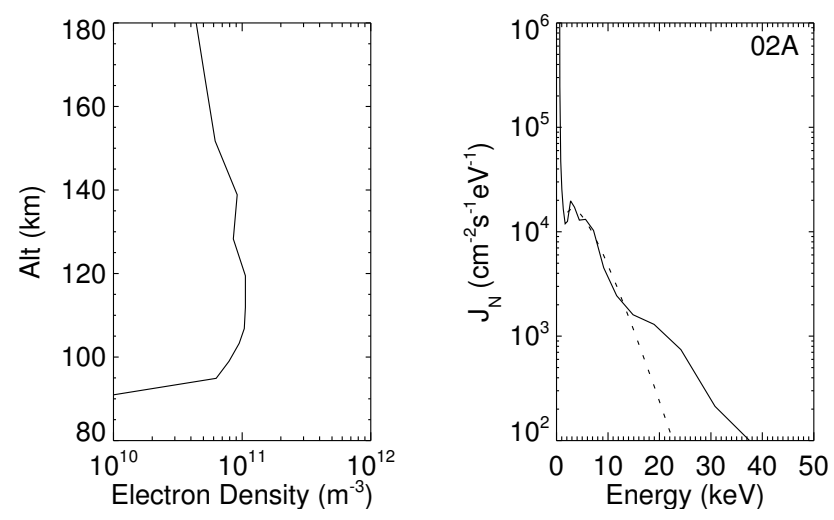

Time: 2002-03-05, 20:13:59 UTC
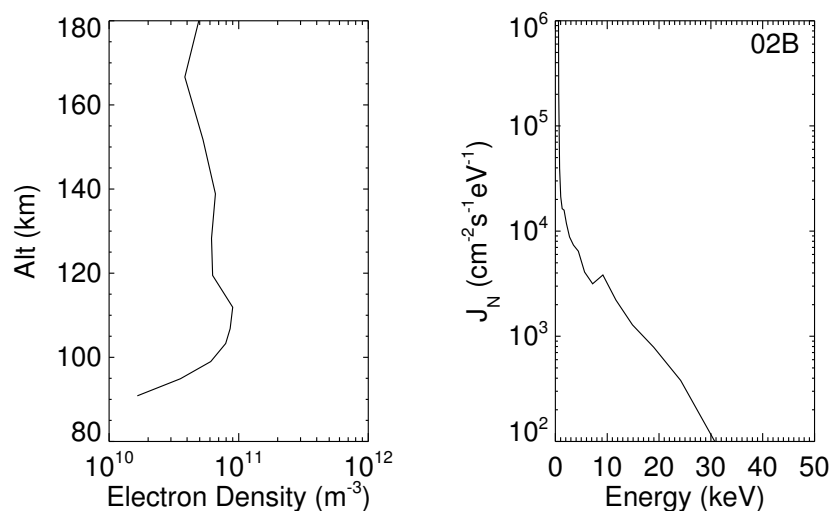

Fig. 7. Electron density profiles and differential number flux (calculated through inversion of the density profile) for events $02 \mathrm{~A}$ and 02B. When applicable, a Maxwellian function (dotted line) has been fitted to the secondary peak in the spectra. This gives approximations to the characteristic energy $\left(E_{0}\right)$ and temperature $(k T)$ of the precipitating electron population.

where $E$ is the energy in $\mathrm{keV}$. This function will not fit the high energy tail so well, but, as mentioned above, the inversion is not so reliable at high energies. Our simpler model will yield a reasonable estimate of the characteristic energy of the suprathermal peaks occurring in the 0.5 to $40 \mathrm{keV}$ range. For $\gamma=0$, Eq. (1) describes an exponential distribution, for $\gamma=1$ a Maxwellian, and for $\gamma \geq 2$ a modified powerlaw distribution (Rees, 1989). $k T$ is the temperature of the source (in $\mathrm{keV}$ ), and we have defined the characteristic energy, $E_{0}$, as the energy for which the supra-thermal distribution peaks. The distribution in Eq. (1) is not an accelerated distribution (Evans, 1974). The reason for this choice is that the electrons generating the diffuse aurora, within which the black aurora appears, are thought not to have been accelerated (Strickland et al., 1994).

The black aurora events studied here last from about 1 to $5 \mathrm{~min}$. As the diffuse aurora changes slowly in each event, and the EISCAT data post-integration time is fairly long (20$30 \mathrm{~s}$ ), the shape of the recovered spectra (Figs. 7 through 10) remains fairly stationary during each of the events. In the cases where a secondary peak is present, the best fit of the 
Time: 2002-03-05, 20:21:59 UTC, $\mathrm{E}_{0}=1.7[\mathrm{keV}], \mathrm{kT}=1.7[\mathrm{keV}]$
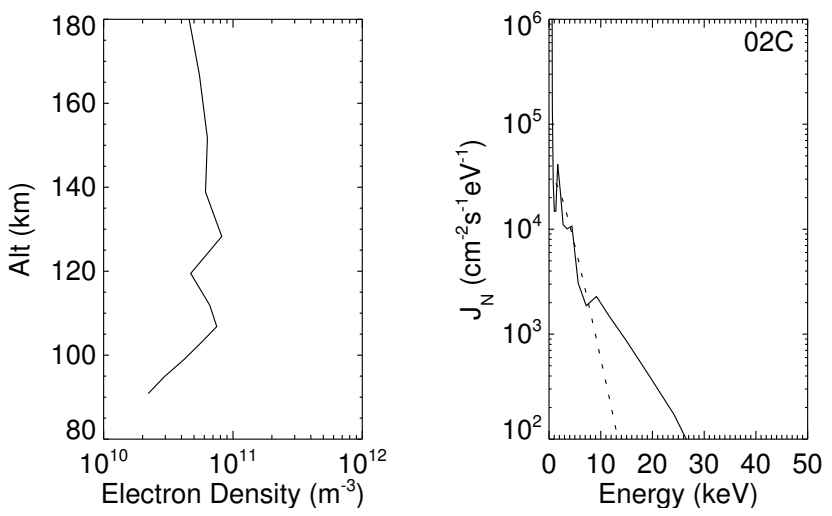

Time: 2003-03-02, 22:02:10 UTC, $\mathrm{E}_{0}=5.5[\mathrm{keV}], \mathrm{kT}=4.9[\mathrm{keV}]$
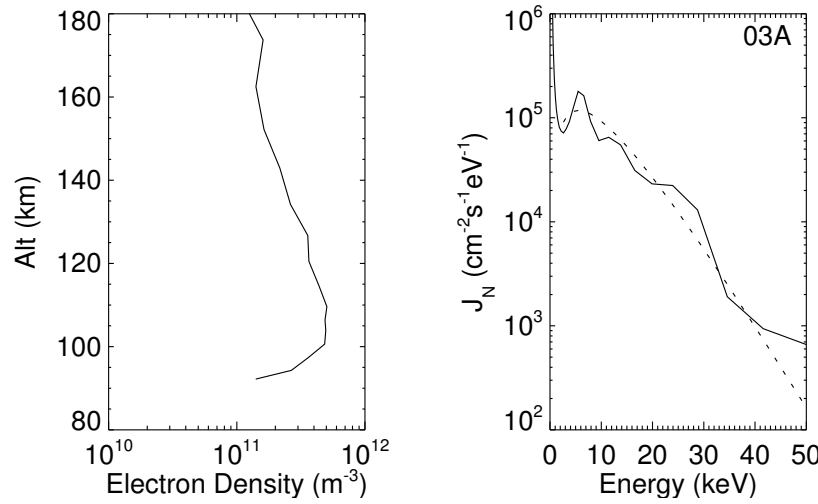

Fig. 8. The same as Fig. 7 but for events $02 \mathrm{C}$ and $03 \mathrm{~A}$.

curve from Eq. (1) is shown as a dotted line. The dotted lines are far from being perfect fits, but we have chosen to use a model with only a few parameters to keep the model simple and transparent. Our major interest is only the location and width of the secondary supra-thermal peak. The resulting characteristic energy ranges from about 2 to $11 \mathrm{keV}$, and Table 4 shows the results (except for $A_{0}$, which does not concern us here) in more detail. Most of the events are quasi-Maxwellian, however, $\gamma=4.0$ in event $03 \mathrm{~B}$ indicates that the energy distribution is more of a modified powerlaw type, which suggests that the tail of the distribution has become wider in this case, presumably under some sort of acceleration process. Figure 11 shows the measured drift velocity of black patches plotted against characteristic energy (shown as asterisks). Note how the drift velocity increases with energy.

\section{Discussion and results}

Our motivation in this paper is to study how the black patches drift relative to the ionospheric plasma, and whether the drift velocity depends on the energy of the electrons in the diffuse aurora. Both of these factors are pertinent to the question of whether the black patches are drifting with the magnetospheric gradient- $B$ curvature drift or not.
Time: 2003-03-02, 22:06:10 UTC, $\mathrm{E}_{0}=9.5[\mathrm{keV}], \mathrm{kT}=2.6[\mathrm{keV}]$
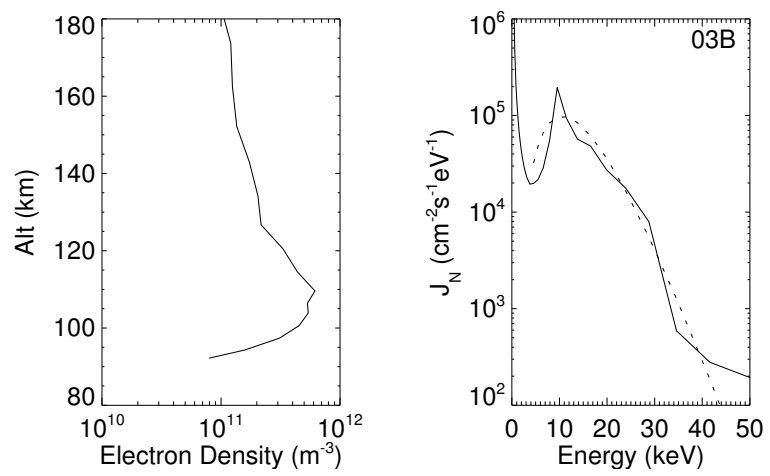

Time: 2003-03-02, 23:56:10 UTC, $\mathrm{E}_{0}=6.6[\mathrm{keV}], \mathrm{kT}=3.8[\mathrm{keV}]$
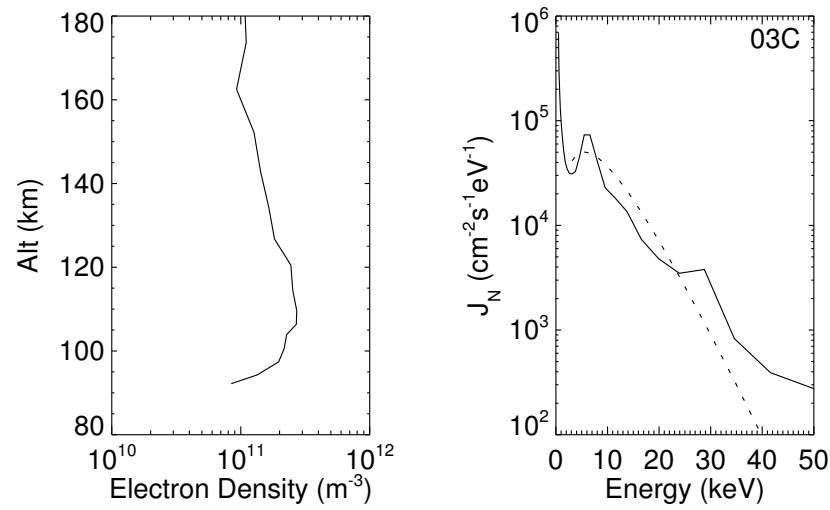

Fig. 9. The same as Fig. 7 but for events 03B and 03C.

As hot plasma convects inwards from the central plasma sheet, the highest energy particles start gradient- $B$ curvature drifting already at $10-12 R_{E}$. Inside of the stable trapping boundary for the high-energy outer belt particles (Feldstein, 1974), there is a relatively warm isotropic plasma $(\sim 1-$ $10 \mathrm{~s} \mathrm{keV}$ ) which is the source plasma for the diffuse aurora (Paschmann et al., 2002). This plasma is bounded on the equatorial side by the $10-100 \mathrm{keV}$ ring current and the $\mathrm{MeV}$ radiation belts. It is safe to say that this source region is bounded between approximately 6 and $12 R_{E}$. It is generally believed that the electron precipitation in the diffuse aurora is due to scattering or diffusion into the loss cone, and not acceleration (Strickland et al., 1994).

Recent results and models suggest that black patches are due to a local drop-out of precipitating electrons, and not due to downward field-aligned current (Kimball and Hallinan, 1998a; Peticolas et al., 2002; Blixt and Kosch, 2004). Kimball and Hallinan (1998a) noted that limited pitch angle diffusion might be responsible for the black patches. They also remarked that the general eastward drift of the observed black patches is consistent with the gradient- $B$ curvature drift of trapped energetic electrons in the magnetosphere, and suggested that the black patches might represent structures in the high-energy electron population, without specifying what these "structures" could be. This model is based on the work by Chiu et al. (1983), who argued that the precipitating hot 
Time: 2003-03-04, 22:58:44 UTC, $\mathrm{E}_{0}=9.5[\mathrm{keV}], \mathrm{kT}=7.6[\mathrm{keV}]$
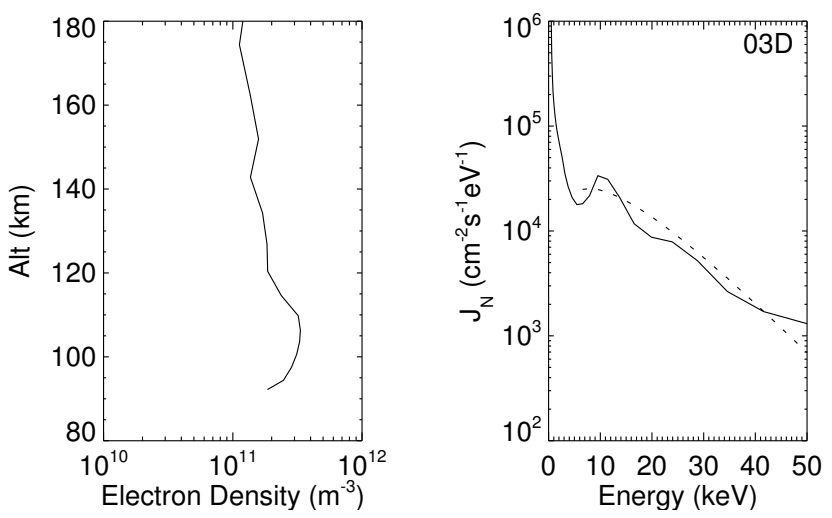

Time: 2003-03-04, 23:10:44 UTC, $\mathrm{E}_{0}=11.5[\mathrm{keV}], \mathrm{kT}=8.5[\mathrm{keV}]$
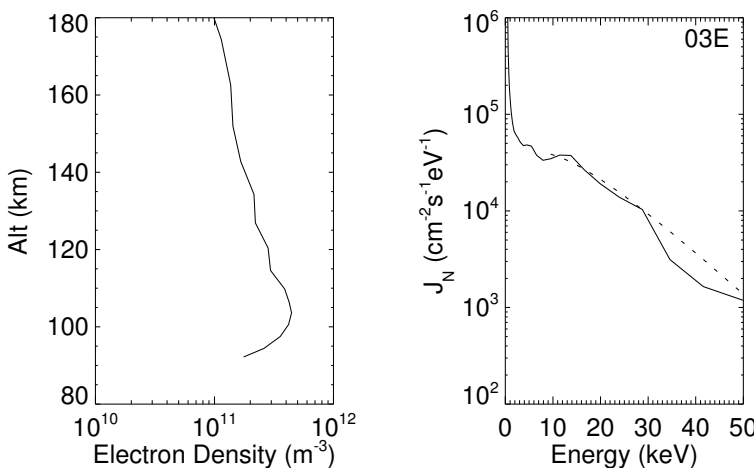

Fig. 10. The same as Fig. 7 but for events $03 \mathrm{D}$ and 03E.

electrons that create optical signatures are gradient drifting azimuthally on trajectories which can depart significantly from the ionospheric convection path. Results from combined FAST and airborne auroral imager studies by Peticolas et al. (2002) indicate (their Fig. 4) that black patches are associated with a large dropout in energy flux at energies of $2-10 \mathrm{keV}$ in the loss cone. They attributed this "missing" loss cone to a local suppression of whistler waves (upperband chorus wave). These waves were assumed to be responsible for the scattering of energetic particle into the loss cone. Furthermore, Blixt and Kosch (2004) showed through EISCAT observations of electron density within non-sheared black patches that they were not associated with any significant downward currents.

Earlier observations indicate that black patches are not drifting with the $\boldsymbol{E} \times \boldsymbol{B}$ flow. Trondsen and Cogger (1997) noticed that black patches drifted with respect to the magnetic field, and not with it. In a group of drifting black patches, individual patches underwent identical deformations at a fixed position in space, suggesting that they might have been drifting relative to a fixed magnetic field. Simultaneous measurements of drifting black aurora and ionospheric electric fields by Schoute-Vanneck et al. (1990) also showed that the black aurora did not drift with the $\boldsymbol{E} \times \boldsymbol{B}$ flow in the ionosphere.

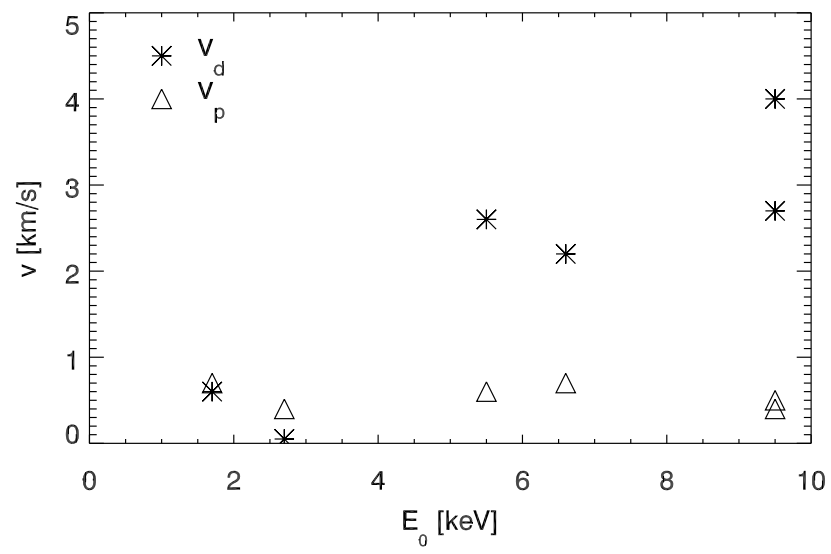

Fig. 11. Scatter plot of the observed black auroral drift $\left(v_{d}\right.$, asterisks) and measured plasma flow velocity ( $v_{p}$, triangles) against the characteristic energy $\left(E_{0}\right)$.

If the black patches are due to structures (where the pitch angle diffusion is locally hindered, or not enabled) in the high-energy electron population, which drift along with the magnetospheric plasma, we should see an energy dependence in the drift of the black patches. However, it should be noted that these localized regions need not drift with the hot plasma, they could drift along with structures in the waves which are responsible for the strong pitch angle scattering, or whatever process is causing the lack of pitch angle scattering in the black aurora. Nonetheless, at this stage we are only capable of testing whether the drift of black patches is consistent with the drift of the hot magnetospheric plasma or with the ionospheric $\boldsymbol{E} \times \boldsymbol{B}$ velocity, as the exact mechanism or process from which the black patches are generated is still unknown. Figure 11 is arranged so as to show if such an energy dependence is present. It shows a scatter plot of the observed drift velocity of the black aurora (asterisks) versus the measured characteristic energy $\left(E_{0}\right)$ of the precipitating particles within the diffuse background aurora, and there is clearly an increase in drift speed with increasing energy. We have used the maximum velocity observed during each event in Fig. 11. Events 02B and 03E are not included (for 02B we have no estimate of the characteristic energy, and for $03 \mathrm{E}$ we have no estimate of the drift velocity of the black aurora). The plot also shows the measured ionospheric plasma flow velocity (triangles) versus $E_{0}$. Note how the plasma flow velocity is essentially the same at all energies. The deduced range of energies fit well with the observations of Peticolas et al. (2002), who associated black auroras with a dropout in electron energy flux at energies of $2-10 \mathrm{keV}$.

We can go a step further and compare the observed drift velocities with the combined gradient- $B$ and curvature drift in the magnetosphere. This drift is given by

$\boldsymbol{v}_{g c}=\frac{\left(W_{\perp}+2 W_{\|}\right)(\boldsymbol{B} \times \nabla) B}{e B^{3}}$,

where $W_{\perp}$ and $W_{\|}$are the perpendicular and parallel energies of the plasma. If the source plasma is considered to be 
isotropic, which is a fairly good approximation in this case, and if the magnetic field is given by a dipole field, which may not be such a good approximation at distances of more than 6 Earth-radii $\left(R_{E}\right)$ away from Earth, the angular velocity of the electrons (eastward in the equatorial plane) is

$v_{\phi}=2 \pi \frac{L W}{3 \times 10^{6}}[\mathrm{rad} / \mathrm{s}]$,

where $L$ is the distance from the surface of the Earth in $R_{E}$, and $W$ is the energy of the electrons in keV (Parks, 1991).

This angular velocity can be mapped down to the ionosphere and the resulting drift velocity in the ionosphere, for different energies and $L$-shells, can be compared with the observed drift velocities of the black patches.

The observed range of velocities $(1.5-4 \mathrm{~km} / \mathrm{s})$ correspond to the gradient- $B$ curvature drift of $10-50 \mathrm{keV}$ electrons for $6 \leq L \leq 12$ (the assumed source region of the diffuse aurora). While these high energies are consistent with the riometer observations of the high energy tail of the precipitating electron energy distribution, it is unlikely that the bulk of the particles generating the diffuse aurora have such high energy. This is foremost because the ionospheric density profile generated by such high energy bulk particles is inconsistent with the density profile measured by the EISCAT radar. The mismatch between observed characteristic energy (2$11 \mathrm{keV}$ ) estimated from the density profiles, and the energy deduced from the gradient- $B$ curvature drift in a dipole field $(10-50 \mathrm{keV})$, may have two major contributing factors. First, since the theoretical drift velocity yields higher velocities for larger gradients in $B$, and the dipole field overestimates the gradient in $B$ compared to a stretched dipole, the electrons may, in reality, move slower than what the model suggests. Thus, we overestimate the energy of the electrons at a given drift speed. In order for the energy of the gradient- $B$ curvature drift in a dipole field to match with the observed energy of the diffuse aurora, we need to assume that the dipole field model is overestimating the energy with a factor of three. Although we can expect large variations from the dipole field at distances close to $10-12 R_{E}$ (the outside boundary of the diffuse aurora source region), especially during disturbed conditions such as those reported here, it is beyond the scope of this article to model the gradient- $B$ curvature drift in more realistic magnetic field models. Second, the mechanism that hinders scattering into the loss cone need not drift with the particle population that generates the diffuse aurora. Thus, if the dipole field model can be assumed to be correct, the mechanism that hinders scattering into the loss cone drifts with a higher energy particle population $(10-50 \mathrm{keV})$, while the diffuse aurora is generated by a $2-11-\mathrm{keV}$ electron population.

The tendency for the black aurora drift speed to increase with the energy of the precipitating electrons, and the fact that the absolute majority of black patches are observed to drift eastwards, lend support to the hypothesis that the mechanism generating the black aurora is drifting with the hot magnetospheric electrons. However, there is one case in which black patches drift westward (beginning of 03A, at around 22:02 UT), counter to the gradient- $B$ curvature drift. All earlier observations of drifting black patches (Parsons and Thomas, 1973; Schoute-Vanneck et al., 1990; Trondsen and Cogger, 1997; Kimball and Hallinan, 1998a) are eastward, and it is uncertain what creates the discrepancy in this case. It could be that the substorm onset just prior to the start of the event (according to SAMNET there is one onset at 21:50 and another at 22:11 UT) caused dramatic changes in the shape of the magnetic field line sufficient to temporarily reverse the drift direction (Li et al., 1998).

\section{Summary and conclusions}

Eight events, each about 1-4 min long, where black auroras have been observed through an image intensified video camera and where the EISCAT radar measured the ionospheric plasma flow and electron density, have been examined in order to study whether black patches are drifting with a velocity consistent with the gradient- $B$ curvature velocity of the hot source plasma or not. The results show that most black patches drift eastward, with $v \sim 1.5-4 \mathrm{~km} / \mathrm{s}$, which is faster than that which Kimball and Hallinan (1998a) and Trondsen and Cogger (1997) observed, but similar to what Parsons and Thomas (1973) and Schoute-Vanneck et al. (1990) observed. The observed drifts of the black patches is several $\mathrm{km} / \mathrm{s}$ faster than the ionospheric plasma flow, but often in the same direction. One case of westward drifting black patches, opposite to the gradient- $B$ curvature drift, is reported for the first time. The reason for this westward drift remains unclear.

Through an inversion of the electron density profiles, the characteristic energy of the precipitating electrons has been estimated, and these agree well with observations by Peticolas et al. (2002). Most significantly, we have found that the drift velocity of the black patches increases with the energy of the precipitating particles. As expected, the $\boldsymbol{E} \times \boldsymbol{B}$ velocity was found to be unrelated to the particle energy. The increase in drift velocity with energy and the direction is consistent with the magnetospheric gradient- $B$ curvature drift, but only an order-of-magnitude agreement in actual drift speed could be established with a rudimentary model of the gradient- $B$ curvature drift speed in a dipole field.

Our findings support the theory that black patches are due to structures in the magnetospheric hot plasma where scattering into the loss cone has been suppressed, and that these structures are gradient- $B$ curvature drifting along with the hot plasma, thereby generating an eastward drift of the black patches which is increasing with increasing energy of the precipitating electrons.

Acknowledgements. The authors would like to thank S. Nozawa and Y. Ogawa for the analysis of the EISCAT data. The EISCAT Scientific Association is supported by Centre National de la Recherche Scientifique of France, Max-Planck-Gesellschaft of Germany, Particle Physics and Astronomy Research Council of the United Kingdom, Norges Forskningsråd of Norway, Naturvetenskapliga Forskningsrådet of Sweden, Suomen Akatemia of Finland and the National Institute of Polar Research of Japan. 
Topical Editor W. Kofmann thanks D. Knudsen and another referee for their help in evaluating this paper.

\section{References}

Blixt, E. and Kosch, M.: Coordinated optical and EISCAT observations of black aurora, Geophys. Res. Lett., 31(L06), 813, doi:10.1029/2003GL019244, 2004.

Brekke, A., Hall, C., and Hansen, T.: Auroral ionospheric conductances during disturbed conditions, Ann. Geophys., 7, 269-280, 1989.

Chiu, Y., Schultz, M., Fennel, J., and Kishi, A.: Mirror instability and the origin of morningside auroral structure, J. Geophys. Res., 88, 4041-4054, 1983.

del Pozo, C., Kosch, M., and Honary, F.: Estimation of the characteristic energy of electron precipitation, Ann. Geophys., 20, 1349-1359, 2002a,

SRef-ID: 1432-0576/ag/2002-20-1349.

del Pozo, C., Williams, P., Gazey, N., Smith, P., Honary, F., and Kosch, M.: Multi-instrument observations of the dynamics of auroral arcs: a case study, J. Atmos. Terr. Phys., 64, 1601-1616, doi:10.1016/S1364-6826(02)00083-4, 2002b.

Evans, D.: Precipitating electron fluxes formed by a magnetic feldaligned potential difference, J. Geophys. Res., 79, 2853-2858, 1974.

Feldstein, Y.: Night-time aurora and its relation to the magnetosphere, Ann. Geophys., 30, 259, 1974.

Haerendel, G., Buchert, S., Hoz, C. L., Raaf, B., and Rieger, E.: On the proper motion of auroral arcs, J. Geophys. Res., 98, 60876099, 1993.

Keskinen, M. and Ganguli, G.: Simulation of dynamics and structure in the black aurora, J. Geophys. Res., 101, 26 995-26999, 1996.

Kimball, J. and Hallinan, T.: Observations of black auroral patches and of their relationship to other types of aurora, J. Geophys. Res., 103, 14671-14 682, 1998a.

Kimball, J. and Hallinan, T.: A morphological study of black vortex streets, J. Geophys. Res., 103, 14 683-14 695, 1998 b.

Kirkwood, S. and Eliasson, L.: Energetic particle precipitation in the substorm growth phase measured by EISCAT and Viking, J. Geophys. Res., 95, 6025-6037, 1990.

Kletzing, C., Scudder, J., Dors, E., and Curto, C.: Auroral source region: Plasma properties of the high-latitude plasma sheet, J. Geophys. Res., 108, 4-1, doi:10.1029/2002JA009678, 2003.

Li, X., Baker, D. N., Temerin, M., Reeves, G. D., and Belian, R. D.: Simulation of dispersionless injections and drift echoes of energetic electrons associated with substorms, Geophys. Res. Lett., 25, 3763-3766, doi:10.1029/1998GL900001, 1998.
Marklund, G., Karlsson, T., and Clemmons, J.: On low-altitude particle acceleration and intense electric fields and their relationship to black aurora, J. Geophys. Res., 102, 17 509-17 522, 1997.

Olsson, A. and Janhunen, P.: Field-aligned conductance values estimated from Maxwellian and kappa distributions in quiet and disturbed events using Freja electron data, Ann. Geophys., 16, 298-302, 1998,

SRef-ID: 1432-0576/ag/1998-16-298.

Osepian, A. and Kirkwood, S.: High-energy electron fluxes derived from EISCAT electron density profiles, J. Atmos. Terr. Phys., 58, 479-487, 1996.

Parks, G.: Physics of Space Plasmas, Addison Wesley, 1991.

Parsons, N. and Thomas, I.: Spatially forbidden regions in the aurora, Can. J. Phys., 51, 1377-1378, 1973.

Paschmann, G., Haaland, S., and Treumann, R.: Chapter 8 - dynamic coupling to the magnetosphere, Space Sci. Rev., 103, 377414, doi:10.1023/A:1023090902586, 2002.

Peticolas, L., Hallinan, T., Stenbaek-Nielsen, H., Bonnell, J., and Carlson, C.: A study of black aurora from aircraft-based optical observations and plasma measurements on FAST, J. Geophys. Res., 107, doi:10.1029/2001JA900157, 2002.

Rees, M.: Physics and Chemistry of the Upper Atmosphere, Cambridge University Press, 1989.

Royrvik, O.: Pulsating aurora: Local and global morphology, Ph.D. thesis, University of Alaska, Fairbanks, 1976.

Schoute-Vanneck, H., Scourfield, M., and Nielsen, E.: Drifting black aurorae, J. Geophys. Res., 95, 241-246, 1990.

Semeter, J. and Kamalabadi, F.: Determination of primary electron spectra from incoherent scatter radar measurements of the auroral E-region, Radio Science, 40, RS2006, doi:10.1029/2004RS003042, 2005.

Strickland, D., Hecht, J., Christensen, A., and Kelly, J.: Relationship between energy flux $Q$ and mean energy $<E>$ of auroral electron spectra based on radar data from the CEDAR Campaign at Sondre Stromfjord, Greenland, J. Geophys. Res., 99, 1946719473, 1994.

Trondsen, T. and Cogger, L.: High-resolution television observations of black aurora, J. Geophys. Res., 102, 363-378, 1997.

Vasyliunas, V.: A survey of low-energy electrons in the evening sector of the magnetosphere with OGO1 and OGO3, J. Geophys. Res., 73, 2839-2884, 1968.

Vondrak, R. and Baron, M.: A method of obtaining the energy distribution of auroral electrons from incoherent scatter radar measurements., in Radar Probing of the Auroral Plasma, (Ed.)Brekke, A., 315-330, Universitetsforlaget, Oslo, Oslo, Norway, 1975. 